3 Fermente nur selten proportional vermehrt erscheinen. Wir dürfen deshalb nicht eines der Fermente zum Maßstab für die Sekretion der Drüse nehmen. Höchstens könnten wir die Partiärfunktionen abschätżen. In diesem Sinne könnte man beachten, daß in Fall I4 auch auf Ätherreiz nur die Diastasewerte gestiegen sind, dagegen fielen die Trypsin- und Steapsinwerte sogar noch niedriger aus. Doch auch hierfür steht es mit der Bewertung der quantitativen Unterschiede schlecht. Wir besitzen noch keine genügende Sicherheit über die starken Schwankungen unterworfenen Normalwerte. v. NoordeN sagt daher, der Ausschlag der Fermentproben sei kein zuverlässiger Maßstab für die Fermentausscheidung. Mit GANG und $K_{L E I N}{ }^{1}$ ) glauben wir, daß man nur starke Herabsetzung oder Fehlen der Fermente klinisch verwerten kann.

Fall 2 und Fall 2o, in denen Durchfälle bestanden, haben übrigens hohe Fermentmengen, so daß wir, (mit Gross u. a.), keinen Parallelismus zwischen Durchfall und Unterfunktion des Pankreas bei unseren Achylikern finden. Akute Ruhrfälle wie Fall 6 scheiden für Erörterung dieser Frage natürlich aus.

Es ergibt sich für uns der Schlu $\beta$, daß wir die gastrogenen Diarrhoen des Achylikers nicht auf eine Pankreasinsuffizienz zurückführen dürfen.

Es scheint nun, daß gelegentlich bei Achylie (vielleicht auch als kompensatorischer Vorgang) reichlich Bauchspeichel gebildet wird (Fall 2, 4, I5, 16), und das würde zu dem Befund passen, der kürzlich in der durch den einen von uns angeregten Dissertation des Zahnarztes GEBIGKE mitgeteilt wurde: daß nämlich in manchen Fällen von Achylia gastrica eine abnorm starke Speichelsekretion vorhanden ist. Es wurde das durch genaue quantitative Messung des fortlaufend $a b-$ gesaugten Speichels festgestellt. Wenn bei manchen Autoren die Neigung besteht, aus Gründen des Ionengleichgewichtes anzunehmen, daß einem Rückgang der Salzsäurebildung im Magen ein Rückgang in der Abscheidung der alkalischen Verdauungssäfte entsprechen müßte, so scheint diese Vorstellung nicht biologisch genug. Die Erhaltung des Ionengleìchgewichts im Blut und im Körper ist ein so komplizierter Vorgang, daß Rückwirkungen der Magendrüsentätigkeit auf Speicheldrüsen und Pankreas nicht ohne weiteres anzunehmen sind.

Man muß gegen die Methode einwenden, daß unser Atherreiz ein unphysiologischer ist und $\mathrm{da}$ wir Pankreassaft vielleicht in Fällen erhalten, in denen eine Herabminderung der Sekretion durch das Fehlen des normalen Salzsäurereizes dennoch vorhanden war. Deshalb erscheint uns die Athermethode zunächst nur geeignet, wirkliche Schädigungen der Saftbildung nachzuweisen, nicht rein funktionelle Hypophylien des Pankreas. Trotz dieser Einschränkung halten wir sie für eine methodische Bereicherung vor allem, weil sie gestattet, schnell, reichlich und fast rein Pankreassekret zu erhalten.

Gegen unsere Ausführungen über die Achylia gastrica gilt der eben angeführte Einwand insofern nicht, als sich unsere Ausführungen über Fehlen einer Pankreasachylie und gelegentliches Vorkommen einer kompensatorischen, pankreatischen Hypersekretion bei Magenanacidität auch auf die Feststellungen stützt, die wir am Duodenalinhalt machten, ehe der Ätherreiz angewandt wurde.

Wir untersuchten auch einige Diabetesfälle, die Befunde veranschaulicht Tabelle B. Bei diesen Fällen sehen wir folgendes:

Bei Fall Nr. I fand sich kein Trypsin im Duodenalsaft, nur nach Ätherspritzung eine kleine Menge. Die Diastasemengen sind dagegen hoch, die Steapsinwerte sehr niedrig. Bei Fall Nr. 2 (schwerer Diabetes) finden sich keine wesentlichen Veränderungen, sogar in einer Verdünnung $r$ : 1o sind die Werte hoch; auch Steapsin nicht vermindert. Fall Nr. 3 zeigte nichts Besonderes, dasselbe gilt für die anderen Fällen, wo vielleicht nur die Diastasemengen bei Verdünnung I : Io etwas niedriger ausfallen.

1) GANG u. KLEIN, Zur klinischen Verwendbarkeit der Doudenalsonde. Med. Klin, I9I4, Nr, 18 v. NOORDEN ${ }^{1}$ ) berichtet darüber, daß er bei einigen Diabetes fällen im Duodenalsaft Verminderung des fettspaltenden Fermentes und des Trypsins fand, auch wenn der Stuhl normal war. Doch weist er eben auf den starken Wechsel dieser Befunde hin. Bei unseren 5 Fällen fand sich nur bei einem eine wesentliche Abnahme des Trypsin- und Steapsingehaltes.

Ob die Reizung des Pankreas durch Äther sich therapeutisch verwerten läßt, steht dahin.

$Z$ u s a $m$ m e $n f$ a s s u n $g$ : 1 . Spritzt man ca. $2-4 \mathrm{ccm}$ Ather durch die Einnornsche Sonde ins Duodenum, so erhält man schnell und reichlich Pankreassaft. Er ist fast rein, enthält daneben nur geringe Mengen Lebergalle. 2. Auch bei funktionellen Hypochylien des Pankreas erhält man auf Ätherreiz reichlich Bauchspeichel. 3. Bei Achylia gastrica ist das Verhalten der Pankreassekretion wechselnd. Neben Verminderungen der Saft- und Fermentproduktion kommen anscheinend auch Vermehrungen: vor. Für die Diarrhoen mancher Achyliker ist nicht die: Unterfunktion des Pankreas bestimmend. 4. Unter 5 Diabetikern zeigte sich nur bei einem im Duodenalinhalt eine wesentliche Minderung des Gehaltes an Trypsin und Steapsin.

\section{ZUR FÖRDERUNG DER RÖNTGENDIAGNOSE DES SUBKARDIALEN ULCUS AN DER KLEINEN KUR- VATUR DURCH DIE LINKE SEITENLAGE.}

Von

Dr. ERICH WolfF, Volontärarzt der Poliklinik.

Aus der Polikinik für Magen- u. Darmkrankheiten von Dr. EMMO SCHLESTNGER.

Die Röntgenuntersuchung des Magens in linker Seitenlage bei Verdacht und Fahndung auf hochsitzende Ulcera ist ein praktisch noch wenig geübtes und nicht hinreichend gewürdigtes Verfahren. EMMO ScHLESINGER machte schon. vor Jahren ${ }^{2}$ ) auf die außerordentlichen Vorzüge dieser Methode an Hand mehrerer einschlägiger Fälle aufmerksam. Inzwischen ist an der hiesigen Klinik eine ganze Reihe von Untersuchungen in linker Seitenlage vorgenommen worden und kurz zusammenfassend muß gesagt werden, daß für die Sicherheit der Diagnose einwandfrei die linke Seitenlage in einer ganzen Reihe von Fällen allen anderen Versuchsanordnungen als überlegen zu betrachten ist. Allein die Tatsache, đaß die Diagnose eines hochsitzenden Ulcus an der kleinen Kurvatur in einer, wenn auch kleinen Anzahl von Fällen, ohne unsere Methode eine Unmöglichkeit gewesen wäre, macht ihre Anwendung im gegebenen Fall zu einem absoluten Erfordernis. Gerade das hochsitzende Ulcus erheischt eine besonders vollkommene Ausnutzung seiner Röntgensymptome, da uns hier die bei pylorusnäheren Geschwüren auftretenden und auf dem Schirm sichtbaren Reflexwirkungen auf die Pförtnertätigkeit nur abgeschwächt entgegentreten und teilweise ganz ausfallen, so daßSpasmus an der großen, Nische an der kleinen Kurvatur, sowie Adhäsionen oft die einzigen Symptome des Röntgenbildes sind, auf die wir rechnen können.

Es ist eine bekannte und allgemein geübte Methode, durch Rechtslagerung des Patienten die bei einer Normalaufnahme gewonnenen Befunde zu erweitern. In analoger Weise soll durch Linkslagerung die spastische Contractur der großen Kurvatur, die bei vertikaler Stellung oberhalb des Füllungsspiegels liegt und so unsichtbar wird, in den Bereich des Kontrastbreies gezogen werden, wobei man gut tut, den Oberkörper ein wenig nach abwärts zu neigen, um die sog. Funduspartic des Magens besonders reichlich $\mathrm{zu}$ füllen und damit den Spasmus sicher zur Darstellung zu bringen.

Die diagnostischen Vorteile, die uns diese Methode bietet, werden am besten durch Wiedergabe folgender Fälle evident:

Fall I. E. R. seit Wochen appetitlos. Es werden nur flüssige Speisen vertragen. Obstipation; starke Abmagerung. Leib stark gespannt; nirgends Druckempfindlichkeit. Ausheberung nach P. F. ergibt normale Werte Ges. Acid. 44 reichlich Schleim, okkultes Blut in den Faeces.

Röntgenuntersuchung am I5.VI. I92I.

1) v. NOORDEN, Die Zuckerkrankheit. 7. Auflage I9I5.

2) Fortschritte auf dem Gebiete der Röntgenstrahlen, Band 25. 
Es zeigt sich bei der Durchleuchtung eine gleichmäßig breite Entfaltung, wobei ebenso wie auf der Platte (s. Abb. I) eine ganz auffallende Verziehung des Magens nach links in die Erscheinung tritt. Caudaler Pol bis anderthalb Handbreite unterdemNabel.Lebhafte Peristaltik, kräftige pylorische Abschnürungen. scheinlich gemacht, jedoch erhalten wir über den Sitz des Ulcus durch diese dorsoventrale Untersuchung ebensowenig weitere Aufschlüsse wie durch die kurz hinterher angestellte Untersuchung in rechter Seitenlage. Dagegen zeigt die Aufnahme bei Linkslagerung (siehe

Abb. 4) eine markante spastische Contractur an der großen Kurvatur.

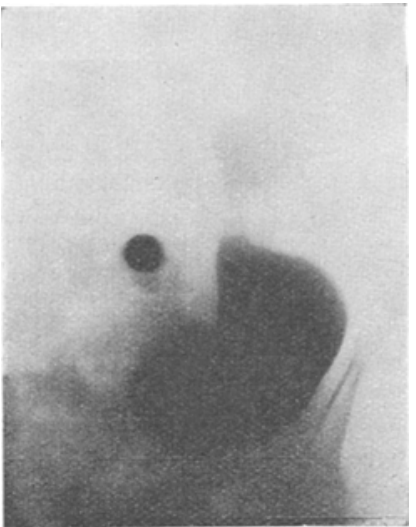

Abb. I. Ulcus an der kleinen Kurvatur im Bereich der Intermediärschicht.

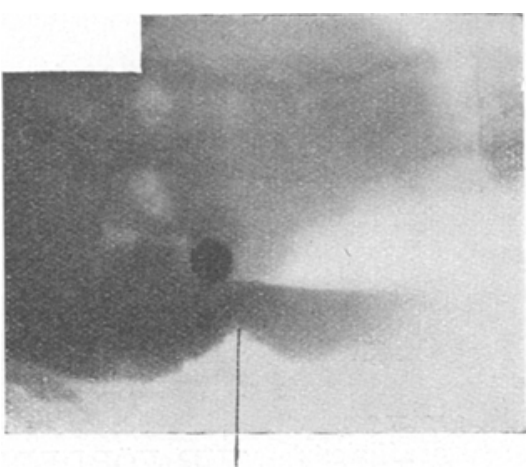

Abb. 2. Derselbe Fall in 1. Seitenlage, der Pfeil zeigt den Spasmus.

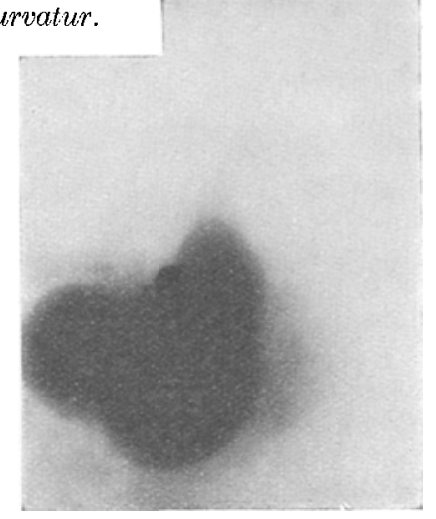

Abb. 3. Hochsitzender Ulcus a, d, kl. Abb. 3. Hochsitzender Ulcus a, d, kl.
Kurvatur im Bereich der Intermediärschicht.

Es bleibt die Frage offen, woraus die Linksverlagerung entspringt. Zweifellos bestehen hier irgendwo adhäsive Veränderungen, womöglich auf ulceröser Basis, denn auch in rechter Seitenlage bleibt der absteigende Schenkel weit links unbeweglich in horizontaler Lage liegen. Außerdem zeigt sich in rechter Lage eine deutliche $Z$ weiteilung der Magenblase, also ein weiterer Hinweis auf Adhäsionen. Durch linke Seitenlage (s. Abb. 2) mit nach abwärts geneigtem Oberkörper wird der Kontrastbrei über den kardialen Teil des Fundus in voller Breite ergossen und nunmehr zeigt sich in typischer Weise eine spastische Einziehung an der großen Kurvatur, so daß wir in der Lage sind, die Diagnose Ulcus ventriculi gegenüber der kontrahierten Stelle stellen zu können.

Fall 2. F. K. Vor anderthalb Jahren längere Zeit wegen Magengeschwürs im Krankenhaus behandelt; ein Jahr darauf Rückfall und zum erstenmal Blutbrechen. Es bestehen an sonstigen Symptomen jetzt sehr heftige vom Magen zum Rücken ausstrahlende Schmerzen, Appetitlosigkeit, Obstipation. Magenausheberung nach P. F.: II $5 \mathrm{ccm}$ stark schleimiger Inhalt, makr. Spuren geronnenen Blutes, Benz. +, Ges. Acid. 48. Auch die Ausheberung des nüchternen Magens zeigte eine positive Benzidinprobe; palpatorisch: deutliche Druckempfindlichkeit" ${ }_{-}$wischen Proc. xyphoideos und Nabel.

Röntgenuntersuchung vom 30 . VI. I92 I.

Die Röntgenuntersuchung, die uns über Sitz und sonstige Besonderheiten des fraglos vorhandenen Ulcus Aufklärung geben soll, zeitigt folgende Resultate: mittelbreitbasige Entfaltung, caudaler Pol handbreit unter dem Nabel, lebhafte Peristaltik. Auffallend ist eine be-

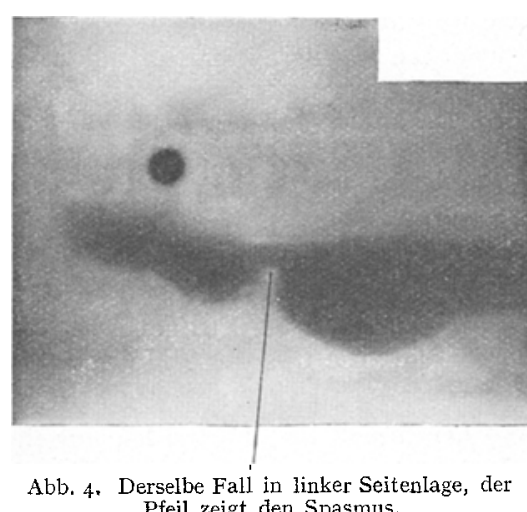

Pfeil zeigt den Spasmus.

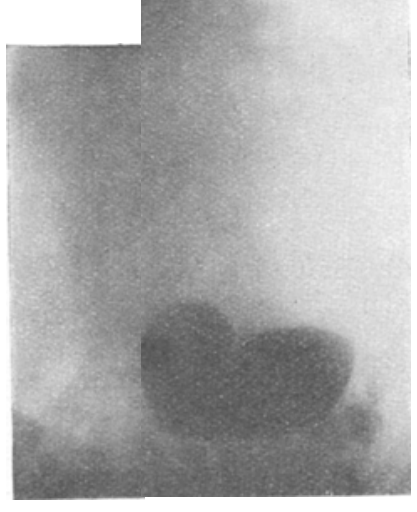

Abb. 5 . Subkardiales Ulcus an der leinen Kurvatur.
Dies Beispiel zeigt klar und deutlich, daß die Untersuchung in linker Seitenlage uns das Röntgenbild eines Ulcus ventriculi vorzuführen imstande ist, wenn alle anderen Versuchsanordnungen versagen. Die Normalaufnahme (Abb. 3) zeigt an der kleinen und großen Kurvatur nicht die geringste Veränderung, welche ein Ulcus vermuten läßt.

Fall 3. A. M. klagt seit ro Jahren über Schmerzen und Kolikanfälle im Magen, und zwar hat er das Gefühl, als ob er während des Essens Luft verschlucke, die sich im Magen ansammelt und seine Beschwerden hervorruft. Wenn er aufstoßen kann, fühlt er Besserung, sonst bleibt scheinbar die nachher aufgenommene Nahrung in der Speiseröhre stecken und verursacht Brechreiz. Jedoch fördert er nur Schleim zutage. Gewichtsabnahme: 60 Pfund. Die andererseits vorgenommene Magenausheberung ergab wechselnde Befunde, sicherlich, weil die Sonde infolge eines intermittierenden Kardiospasmus (s. u.) bisweilen an der Kardia steckenblieb, bisweilen in den. Magen gelangte.

Die Durchleuchtung (I 5. IX. I92I) der Speiseröhre zeigte nämlich eine weitgehende Stauung oberhalb der Kardia, denn noch nach dreiviertel Stunden isr der Ösophagus ganz mit Brei gefüllt und erst nach Einnahme eines Glases Wasser gleitet der Brei in den Magen

Röntgenuntersuchung am I7. IX. I921.

Die Röntgenuntersuchung des Magens ergibt in dorsoventraler Richtung (s. Abb. 5) eine Lage des caudalen Pols 3 Querfinger unterhalb des Nabels; es ist nur der unterste Teil des ${ }^{\top}$ Magens sichtbar. Irgendwelche diagnostische Schlüsse läßt dieses Bild nicht zu. Anders liegt es schon bei der Betrach-

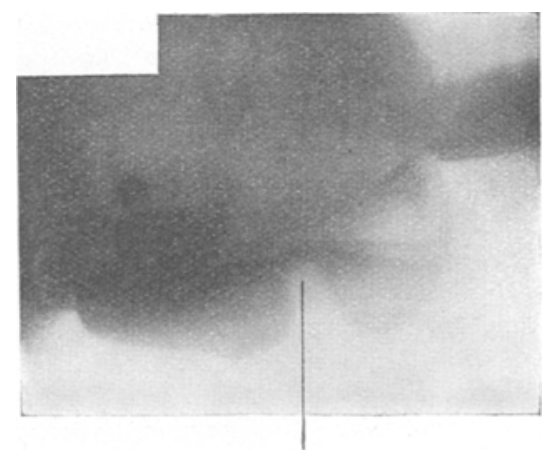

Abb. 6. Derselbe Fall in linker Seitenlage.

tung der in rechter. Seitenlage gewonnenen Platte. Wir finden einen guten Abfall des Pylorus nach rechts, während Kardia und Anfangsteil des Korpus sichtlich adhärent erscheinen, so daß wir Verdacht auf anatomische Veränderungen sonders auf der Platte (s. Abb. 3) zutage tretende Rechtsverziehung sowohl des absteigenden Schenkels als auch des Pylorus. Verweildauer I2-I6 Stunden. Wir finden also durch die Röntgenuntersuchung unsere Diagnose wahr- 
dieser Gegend schöpfen müssen, der noch außerdem in dem Auftreten einer kleinen, nur schwach sichtbaren Luftblase an der kleinen Kurvatur in demselben Bezirk Nahrung findet. - Es ist gewiß nicht immer sicher zu sagen, inwieweit man bei der Entdeckung so geringer Helligkeitsunterschiede, als welche sich kleine Luftblasen auf der Röntgenplatte repräsentieren, subjektiven Einflüssen unterworfen ist; und die Gefahr, mehr zu sehen, als das Bild wirklich zeigt, besteht gerade in nicht ganz eindeutigen Fällen in erheblichem Maße; umso mehr müssen wir alle uns zu Gebote stehenden Wege beschreiten, ehe wir unsere Diagnose endgültig festlegen. So wurde auch hier die linke Seitenlage (s. Abb. 6) in Anwendung gebracht und unser Verdacht auf ulceröse Veränderungen an der kleinen Kurvatur im oberen Bereich des Korpus findet durch das Auftreten einer scharfen, spastischen Contractur gerade gegenüber der oben erwähnten in rechter Seitenlage zu sehenden Luftblase volle Bestätigung. Wie schwer im ganzen die anatomischen Veränderungen dieses Falles, die sich bei dorsoventraler Stellung und Rechtslagerung verhältnismäßig wenig dokumentieren, in Wirklichkeit sind, zeigt besonders deutlich die Aufnahme in Rückenlage (s. Abb. 7), wo wir eine starke, sanduhrförmige Einschnürung dicht unterhalb der Kardia mit einer Ausbuchtung ähnlich einem Ulcus penetrans und eine ganz dichte Einziehung an der großen Kurvatur zu sehen bekommen.

Gerade dieser letzte Fall rückt den Wert der linken Seitenlage in ganz besonderes Licht. Es ist uns heute zur Genüge bekannt, daß diejenigen Fälle von Magenulcus, die anamnestisch und klinisch vollkommen atypisch sind und sich bei der Röntgendurchleuchtung einwandfrei als Geschwür dokumentieren, keinesfalls zu den Seltenheiten gehören. Wie sich Derselbe Fall in Ruickenlage, der Pfeil zeigt die subkard. sanduhrförmige Einziehung.

die Röntgendiagnostik ohne Zweifel einen hervorragenderen und wesentlicheren Anteil im Laufe der Jahre erobert hat, als man ihr ehedem zuerkannte, so kann sie uns doch in vielen Fällen eben nur dann zum Ziel führen, wenn wir alle Möglichkeiten, die sie uns bietet, voll ausnutzen und uns mit schematisierten Versuchsanordnungen nicht zufrieden geben. Von diesem Gesichtspunkt aus ist es unerläBlich, die Magenuntersuchung in linker Seitenlage in weitaus größerem Umfange, als es bisher der Fall war, als Untersuchungsmethode einzuführen und ihr den Platz in der Technik der Röntgendiagnostik einzuräumen, der ihr zukommt. Die Erfolge bei mehr als einem Dutzend von Fällen, die in letzter Zeit an der hiesigen Klinik und Poliklinik auf diese Weise erzielt wurden, geben uns das Recht, erneut auf sie aufmerksam zu machen und für sie einzutreten.

\section{DER BLUTZUCKERSPIEGEL NACH INTRAVENÖSEN INFUSIONEN HOCHPROZENTIGER TRAUBEN- ZUCKER-LÖSUNGEN BEIM KINDE.}

Von

HANS OPITZ.

Aus der Universitäts-Kinderklinik Breslau. (Direktor: Prof. Dr. STOLTE.)

Die biologische Wirkung intravenöser Traubenzuckerinfusionen ist in den letzten Jahren verschiedentlich bei Tieren wie bei Menschen studiert worden. Meist wurden relativ niedrige Konzentrationen (bis 25 proz. Lösungen) verwandt. U'ber die Verabfolgung sehr konzentrierter Zuckerlösungen beim Menschen äußern sich, soweit ich die Literatur übersehe, bei Erwachsenen nur BürGER und HAGEMANN sowie Korbsch, bei Säuglingen BEUMER ${ }^{1}$ ). Nur der letztgenannte, der 70 proz. Lösungen injizierte, macht uns Angaben über den Ablauf der Blutzuckerkurve. KorBsch berichtet kurz über die klinische Wirkung 5o proz. Glykoselösungen, während BÜRGER und HAGEMANN in eingehenden Untersuchungen in erster Linie die osmotischen Verhältnisse nach 35 proz. bis mehr als 50 proz. Traubenzuckerinfusionen verfolgten.

Die Vergleichung der. Resultate der verschiedenen Autoren wird dadurch erschwert, daß die einen offenbar die Konzentration der Lösungen nach Gewichts-, die anderen nach Volumenprozenten angeben. Letzteres muB der Fall sein, wenn man von fast roo proz. Lösungen (BEUMER) spricht. Der Unterschied ist sehr beträchtlich, wie folgendes Beispiel zeigt:

Setzt man zu 4,5 g Dextrose zehntelkubikzentimeterweise destilliertes. Wasser, so sind $1,5 \mathrm{ccm}$-erforderlich, um eine zwar dicksirupöse aber klare Lösung zu erhalten, aus der auch beim Abkühlen auf Körpertemperatur kein Zucker mehr auskristallisiert. Diese würde eine Konzentration von 75 Gewichtsprozenten besitzen. Dagegen nehmen diese $4,5 \mathrm{~g}$ Dextrose $+\mathrm{I}, 5 \mathrm{~g}$ Aq. dest. ein Volumen von noch nicht ganz $4 \mathrm{ccm}$ ein, mit anderen Worten: in $100 \mathrm{ccm}$ dieser Lösung würden $\mathrm{r} 12,5 \mathrm{~g}$ Zucker enthalten sein, entsprechend einer Konzentration von I I 2,5 Volumenprozenten, wobei fehlerhafterweise Gewicht zu Volumen in Beziehung gebracht wird. Einzig exakt ist demnach nur die Angabe in Gewichtsprozenten. Für intravenöse Traubenzuckerinfusionen dürfte eine 75 proz. Lösung die höchste Konzentration darstellen, die man ohne Gefahr anwenden darf.

Was das Verhalten des Blutzuckerspiegels nach intravenöser Zuckerzufuhr anlangt, so wissen wir aus den bisher vorliegenden Untersuchungen, daß sich dieser unmittelbar nach der Infusion sehr steil erhebt, um sehr rasch wieder abzufallen: Thannhauser und Pfitzner, die $500 \mathrm{ccm} 7$ proz. Dextroselösung im Verlauf von I5 Minuten verabfolgten, fanden bereits eine Viertelstunde später wieder normale Werte, desgleichen RYSER, der in der gleichen Zeit $500 \mathrm{ccm}$ 6 proz. Lävuloselösung injizierte, und LOEw> nach Injektion von $200 \mathrm{ccm} 20 \mathrm{proz}$. Traubenzuckerlösung, während NoNNENBRUCH und SZYSZKA, die 30-60 g Glykose in I $5-20$ p:oz. Lösung während 20-55 Minuten intravenös beibrachten, den Blutzuckerspiegel erst nach $1 / 2$ - I Stunde das frühere Niveau erreichen sahen. Mit diesen bei Erwachsenen erhobenen Befunden stimmen die von Beumer bei Säuglingen gewonnenen Resultate überein. Bei Injektionen von nicht mehr als I,2 g pro $\mathrm{kg}$ Körpergewicht war nach 12 Minuten die Hyperglykämie geschwunden, während sie sich bei Mengen von über $2 \mathrm{~g}$ pro $\mathrm{kg}$ noch nach 40 Minuten nachweisen ließ. Im Verlaufe der dem Abfall der Blutzuckerkurve folgenden Stunden fand CHRIsTOFFEL bei Kaninchen einen zweiten steilen aber niedrigeren Gipfel, eine Beobachtung, die MehlHorn und Frank nach peroraler Zuckerzufuhr vielfach bestätigen konnten.

Für das Kindesalter liegen keine Untersuchungen über den Blutzuckerspiegel nach intravenösen Zuckerinfusionen vor, und der ungewöhnliche Ablauf der Blutzuckerkurve bei einem II jährigen Knaben, der wegen Herzinsufficienz infolge von Pericarditis $100 \mathrm{~g}=70 \mathrm{ccm}$ einer 50 proz. Dextroselösung erhalten hatte, veranlaßte uns, die Glykämie bei diesem Patienten genauer zu verfolgen unter Anstellung vergleichender Untersuchungen bei einigen herzgesunden Kindern. Die Analysen wurden mit Hilfe der Bangschen Mikromethode ausgeführt.

Dem erwähnten Patienten Karl Z. waren $50 \mathrm{~g}$ Dextrose in 50 proz. Lösung injiziert worden, d. h. I,786 g pro $\mathrm{kg}$ Körpergewicht, eine Menge, die wir auch allen andern Infusionen zugrunde legten. Wie aus der Kurve von Nr, I hervorgeht, war der Blutzucker nach I Stunde noch ein wenig erhöht, um dann im Verlauf von weiteren $2^{1 / 4}$ Stunden kontinuierlich anzusteigen. Der Kürze wegen sind die Untersuchungsresultate in einer Tabelle und 2 Kurventafeln zusammengestellt. Die nicht registrierten Kurven von Nr. 5

1) Zeitschr. f. Kinderheilk. 29, 352. Iy2r. Literaturverzeichnis siehe dort sowie bei NONNENBRUCH und SZYSZKA, Arch, f, exp. Pathol. u. Pharmakol, 86, 28r. Igzo. 